\title{
Characteristics of Atmospheric Kinetic Energy Spectra during the Intensification of Typhoon Lekima (2019)
}

\author{
Hepeng Zheng ${ }^{1}$, Yun Zhang ${ }^{1, *}$, Yuan Wang ${ }^{1, *} \mathbb{D}$, Lifeng Zhang ${ }^{1}{ }^{\mathbb{D}}$, Jun Peng ${ }^{1}$, Saisai Liu ${ }^{2}$ and \\ Aibing $\mathbf{L i}^{3}$ \\ 1 College of Meteorology and Oceanography, National University of Defense Technology, \\ Nanjing 211101, China; zhenghepeng17@nudt.edu.cn (H.Z.); zhanglif_qxxy@aliyun.com (L.Z.); \\ pengjun@nudt.edu.cn (J.P.) \\ 2 Unit 31010, People's Liberation Army, Beijing 100081, China; liusaisai_2014@sina.com \\ 3 Unit 96941, People's Liberation Army, Beijing 102208, China; cmo_nudt@163.com \\ * Correspondence: zhangyun17@nudt.edu.cn (Y.Z.); wangyuan19@nudt.edu.cn (Y.W.)
}

Received: 30 July 2020; Accepted: 28 August 2020; Published: 31 August 2020

\begin{abstract}
The intensification of Typhoon Lekima (2019) is simulated with the Weather Research and Forecasting model to study the atmospheric horizontal kinetic energy (HKE) spectra and corresponding spectral HKE budgets under the control of real tropical cyclone (TC). The results show that the TC has the ability to modify the canonical atmospheric energy spectrum during its evolution, which is dominated by its rotational mode. With the intensification of Lekima, the HKE spectrum in the troposphere swells over the central mesoscale and develops an arc-like shape. The stronger the $\mathrm{TC}$, the more pronounced the arc-like shape is and the smaller scale it extends to. The roles various physical processes play at different heights and horizontal scales during the intensification of Lekima are investigated and the dependence of the effect of physical processes on scale and height is revealed. Meanwhile, the potential relationship between the intensification of TC, the activation of energy activity at smaller scales, and the downscale extension of the arc-like spectral shape is found.
\end{abstract}

Keywords: tropical cyclone; horizontal kinetic energy spectrum; spectral energy budget

\section{Introduction}

For the past few decades, despite large improvement in the track forecast of tropical cyclone (TC), there is almost no substantial improvement in the intensity forecast for all lead times [1]. Model skill in predicting TC formation and rapid intensification is still very limited [2]. One reason why TC intensity is so poorly predicted lies in that, compared to track, which is more dependent on the environmental conditions, TC intensity can be more strongly dependent on internal dynamics and moist convection, which occur on smaller scales, and are more chaotic, less well understood, and intrinsically less predictable [3]. In this context, a better understanding of TC internal dynamics associated with its intensity variation is essential for improving intensity forecasts $[4,5]$.

Among various methods, energy spectrum analysis is known to reveal signals at different scales. It is thus particularly suitable for investigating physical processes associated with $\mathrm{TC}$, which is known as a typical multiscale system. Atmospheric energy spectrum refers to the distribution (i.e., variance) of atmospheric energy with wavenumber and/or frequency, which can intuitively reflect the scale characteristics of energy. Since the pioneering work of Nastrom and Gage [6], based on data from aircraft flights, many researchers have examined the horizontal wavenumber $\left(k_{h}\right)$ spectrum of the atmospheric energy, especially for the mesoscale range. Numerous observational studies have reached a consensus - the atmospheric horizontal kinetic energy (HKE) spectrum as a function of $k_{h}$ possesses robust canonical shape, which follows a $k_{h}^{-3}$ power law at the synoptic scale and a $k_{h}^{-5 / 3}$ power law at 
the mesoscale, with a transition scale between these two power-law dependence around horizontal wavelength $\left(\lambda_{h}=2 \pi / k_{h}\right)$ of $\sim 500 \mathrm{~km}[7,8]$. Up to now, the underlying mechanism for the formation of the canonical shape of atmospheric energy spectrum, especially for the mesoscale $-5 / 3$ spectral slope, is still under explored [9-12]. Although the mechanism is not clear, the observed atmospheric spectrum has already been reproduced by many numerical models and even becomes an indicator for verifying models [13-16]. In addition to studying the pattern of the energy spectrum, the contributions of various physical processes at different scales to the energy budget to the atmosphere can also be investigated by means of spectral energy budget formulation. As it is difficult for observational methods to provide the comprehensive data needed for budget diagnosis, the relevant studies are always performed based on results from high-resolution numerical simulation [17-23].

Wang et al. [22] (hereafter WZ2018), to our knowledge, were the first to investigate the energy spectrum associated with TC using high-resolution numerical model. Using an idealized simulation, they studied the distribution of mesoscale HKE spectrum and corresponding spectral budget as a function of $k_{h}$ at different vertical heights during the intensification and mature stage of TC, and revealed the specific roles of various physical processes, such as energy cascade, buoyancy effect, convection, and vertical propagation of inertia-gravity waves (IGWs). In particular, they found that unlike the canonical atmospheric spectrum, the HKE spectrum in their simulation displays an arc-like shape at the mesoscale in the troposphere.

Although they have achieved some conclusions, the research of WZ2018 was entirely based on idealized simulation, which suffered lots of simplification. For example, the simulation is performed on the $f$ plane, which does not take the real geography and terrain into account; the initial vortex is artificial and assumed to be axisymmetric; the background atmosphere is specified as motionless, which does not contain any system except for the initial vortex; and the radiation processes are neglected. It is still doubtful to what extent do their conclusions apply to TCs in the real atmosphere, and is essential to carry out relevant research on real cases. Thus, in this study, we examine the characteristics of atmospheric HKE spectra associated with Typhoon Lekima (2019), which formed in August 2019, using the Weather Research and Forecasting (WRF) model [24]. The remainder of the paper is organized as follows. Section 2 provides a brief description of Typhoon Lekima, model configuration, and simulated results. Section 3 describes the spectral HKE budget formulation, and presents the detailed analysis of HKE spectra and diagnosis of spectral HKE budgets during the intensification of Lekima. Discussion and conclusions are given in Section 4.

\section{Numerical Experiment}

\subsection{Typhoon Lekima}

Typhoon Lekima formed in the Northwestern Pacific at around 0900 UTC 4 August 2019, moved northwestward and subsequently experienced an intensification at sea. After developing into a super Typhoon at around 1500 UTC 7 August, Lekima entered the mature stage, which persisted to around 1800 UTC 9 August. It then made landfall in Zhejiang, China, moved northward and began to weaken continuously. According to the best-track data of Japan Meteorological Agency (JMA), Lekima reached its maximum intensity at around 1200 UTC 8 August, with a lowest minimum sea level pressure of $\sim 925 \mathrm{hPa}$ and a highest $10-\mathrm{m}$ maximum wind speed of $\sim 54 \mathrm{~m} \mathrm{~s}^{-1}$.

\subsection{Experimental Design}

In this study, we perform a 48-h numerical simulation of Lekima from 0000 UTC 6 August to 0000 UTC 8 August, which involved the period of primary intensification and initial maturity of the Typhoon. The model used here is the Advanced Research version of WRF (ARW-WRF) model, version 3.6. The model domain is duplicately nested through two-way nesting, and the outer domain is centered at $\left(20.75^{\circ} \mathrm{N}, 127.55^{\circ} \mathrm{E}\right)$ and the inner domain is set to move to keep the simulated TC always locating at the center of the domain. The horizontal grid-point number of the outer and inner domain 
is $601 \times 601$ and $401 \times 401$, with a horizontal resolution of 7.5 and $2.5 \mathrm{~km}$, respectively. Both model domains have 51 vertical levels, with the top at $25 \mathrm{hPa}(z=\sim 25 \mathrm{~km})$.

For both domains, the simulation uses the Thompson microphysics scheme [25], the Yonsei University (YSU) boundary layer scheme [26], and the Rapid Radiative Transfer Model for general circulation models (RRTMG) longwave and shortwave radiation scheme [27]. Rayleigh damping is applied to the vertical velocity in the upper $5 \mathrm{~km}$ of the model domains to prevent artificial reflection of gravity waves from the model top [28]. In addition, the Kain-Fritsch cumulus parameterization scheme [29] is used in the outer domain. To provide the initial fields and lateral boundaries for the simulation, the European Center for Medium-Range Weather Forecasts (ECMWF) ERA5 reanalysis data at a horizontal resolution of $0.25^{\circ} \times 0.25^{\circ}$ and a time interval of $6 \mathrm{~h}$ are used. All analyses in this study are conducted based on the results from the inner domain, which are output at 1-h interval.

\subsection{Overview of the Simulation}

The simulated track, minimum sea level pressure, and maximum 10-m wind speed of Lekima are compared with the best-track data of JMA at 6-h resolution in Figure 1. It can be found that the simulated track of Lekima agrees well with the observation throughout the simulation (Figure 1a). The model reproduces well the intensification of Lekima during the simulated period, and achieves a lowest minimum sea level pressure of $938.8 \mathrm{hPa}$ and highest 10-m maximum wind speed of $48.5 \mathrm{~m} \mathrm{~s}^{-1}$ (Figure 1b).
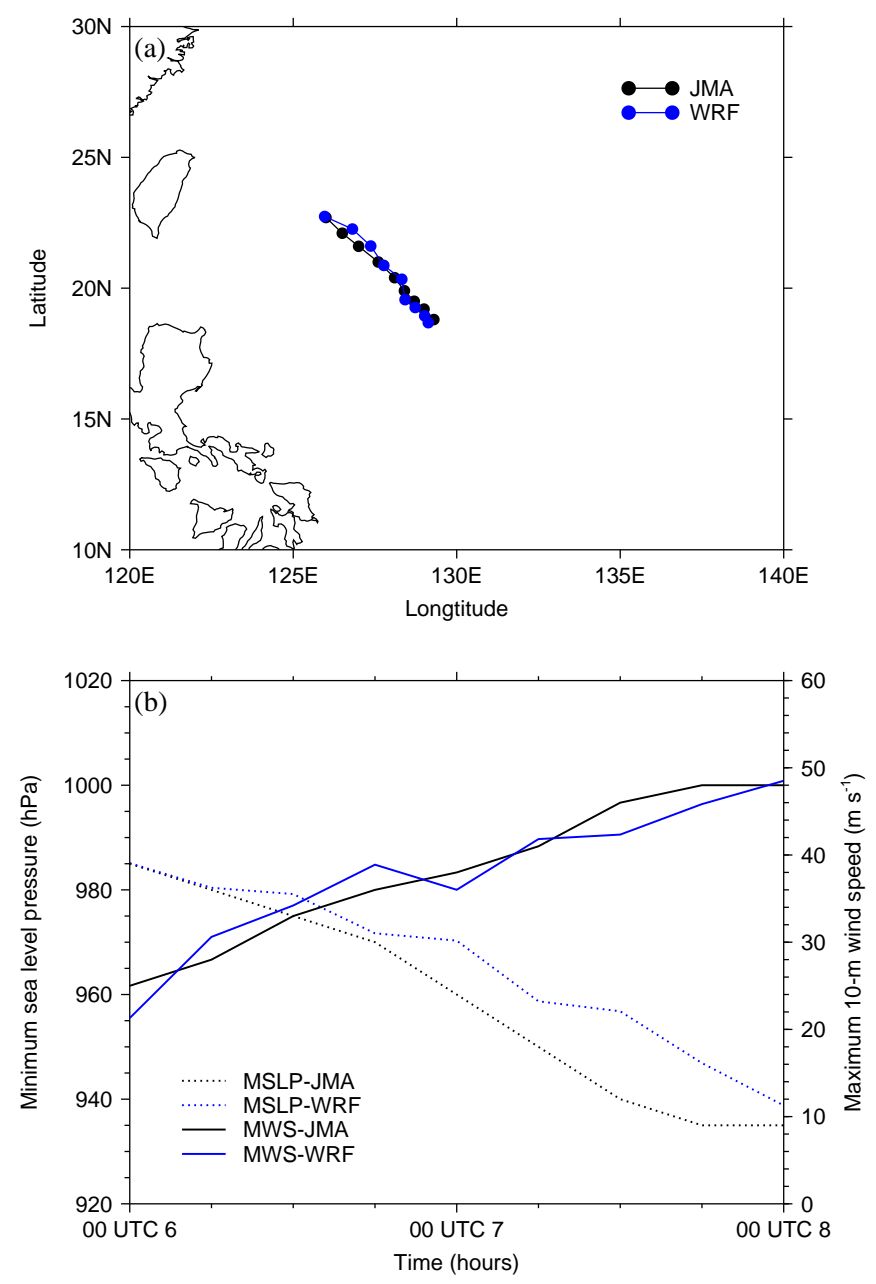

Figure 1. (a) Typhoon track and (b) minimum sea level pressure (MSLP; dotted line) and maximum 10-m wind speed (MWS; solid line) in the JMA observation (black) and simulation (blue). 
Figure 2a shows the vertical profile of the basic-state Brunt-Väisälä frequency during the simulation, which is defined as the inner domain-averaged value and reflects the static stability of the background atmosphere. The frequency is almost constant below $z=5 \mathrm{~km}$, with a mean value of $1.26 \times 10^{-2} \mathrm{~s}^{-1}$. It then decreases slowly from $1.28 \times 10^{-2} \mathrm{~s}^{-1}$ at $z=5 \mathrm{~km}$ to $6.48 \times 10^{-3} \mathrm{~s}^{-1}$ at $z=14 \mathrm{~km}$, while experiences a sharp growth from $z=14 \mathrm{~km}$ and achieves a maximum value of $2.91 \times 10^{-2} \mathrm{~s}^{-1}$ at $z=18 \mathrm{~km}$. Thus, the heights from 14 to $17 \mathrm{~km}$ can be roughly treated as a transition zone from troposphere to stratosphere.
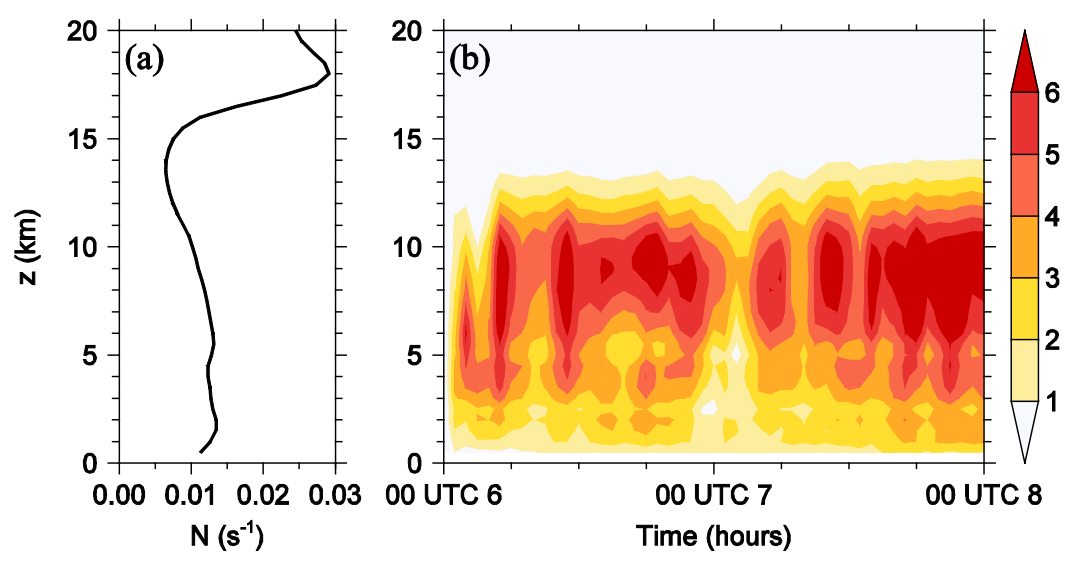

Figure 2. (a) Profile of the basic-state Brunt-Väisälä frequency and (b) time-height distribution of the diabatic heating rate averaged over the main convective region $\left(\mathrm{K} \mathrm{h}^{-1}\right)$.

The diabatic heating released by the microphysical processes can be output by the WRF model directly. The time-height distribution of the heating rate averaged over a $200 \times 200 \mathrm{~m}^{2}$ square horizontal area being centered in the Typhoon is shown in Figure 2b. During the simulation, the powerful heating always penetrates the whole troposphere, implying the vigorous deep convection throughout the intensification of Lekima. In addition, the heating does not distribute uniformly in the troposphere, but exhibits two relatively separated vertical subranges, with heights of 2-5 and 6-12 km, respectively. The lower subrange stems mainly from the release of heating by the condensation process; the upper subrange stems mainly from the release of heating by the condensation, freezing, and sublimation processes; and the gap of heating between these two subranges comes from the absorption of heating induced by the melting process (not shown).

\section{HKE Spectra and Spectral Energy Budgets}

\subsection{Methodology}

In WZ2018, they calculated the energy spectra using the two-dimensional (2D) discrete Fourier transform (DFT). However, using the DFT on regional domains with an aperiodic structure will result in the aliasing of large-scale variance into shorter scales; thus, destroying all usefulness of spectra at large wavenumbers; while the method of discrete cosine transform (DCT) can solve this problem effectively [30]. Thus, in this study, HKE spectra and various spectral budget terms are calculated based on the 2D DCT of fields over the inner domain. Let $\hat{\varphi}(\mathbf{k})$ be the DCT of field $\varphi(x, y)$, where $\mathbf{k}=\left(k_{x}, k_{y}\right)$ is the horizontal wave vector. The HKE spectrum per unit volume is defined as

$$
E_{h}(\mathbf{k})=\bar{\rho}[\hat{\mathbf{u}}(\mathbf{k}) \cdot \hat{\mathbf{u}}(\mathbf{k})] / 2=\bar{\rho}[\hat{u}(\mathbf{k}) \hat{u}(\mathbf{k})+\hat{v}(\mathbf{k}) \hat{v}(\mathbf{k})] / 2
$$

where $\bar{\rho}$ is the height-variant basic-state density and $\mathbf{u}=(u, v)$ is the horizontal velocity vector. 
The HKE spectrum can be further decomposed into horizontally rotational and divergent components $\left[E_{R}(\mathbf{k})\right.$ and $\left.E_{D}(\mathbf{k})\right]$, respectively [18], which are defined as

$$
E_{R}(\mathbf{k})=\bar{\rho} \hat{\zeta}(\mathbf{k}) \hat{\zeta}(\mathbf{k}) /\left(2|\mathbf{k}|^{2}\right) \text { and } E_{D}(\mathbf{k})=\bar{\rho} \hat{\delta}(\mathbf{k}) \hat{\delta}(\mathbf{k}) /\left(2|\mathbf{k}|^{2}\right)
$$

where $\zeta=\partial v / \partial x-\partial u / \partial y$ is the vertical vorticity and $\delta=\partial u / \partial x+\partial v / \partial y$ is the horizontal divergence.

Following Peng et al. [31], the spectral HKE budget formulation is given by

$$
\begin{aligned}
\partial E_{h}(\mathbf{k}) / \partial t= & t_{h}(\mathbf{k})+\partial_{z} F_{h \uparrow}(\mathbf{k})+\partial_{z} F_{p \uparrow}(\mathbf{k})+C_{A \rightarrow h}(\mathbf{k}) \\
& +\operatorname{Div}_{h}(\mathbf{k})+H_{h}(\mathbf{k})+J_{h}(\mathbf{k})+D_{h}(\mathbf{k})
\end{aligned}
$$

where $\partial E_{h} / \partial t$ is net tendency term of HKE; $t_{h}$ is spectral transfer term due to nonlinear interactions; $F_{h \uparrow}$ and $F_{p \uparrow}$ are HKE vertical flux and pressure vertical flux, respectively, and $\partial_{z} F_{h \uparrow}$ and $\partial_{z} F_{p \uparrow}$ are the vertical divergence of the corresponding vertical flux; $C_{A \rightarrow h}$ represents the spectral conversion of available potential energy (APE) to HKE; $\mathrm{Div}_{h}$ is the spectral tendency due to the three-dimensional (3D) divergence; $H_{h}$ is the spectral tendency due to diabatic processes; $I_{h}$ is the adiabatic nonconservation term; and $D_{h}$ is the dissipative term.

The detailed expressions for the terms in Equation (3) are written as

$$
\begin{aligned}
& t_{h}(\mathbf{k})=-\bar{\rho} \hat{\mathbf{u}} \cdot \operatorname{DCT}(\mathbf{u} \cdot \nabla \mathbf{u}+\mathbf{u} \nabla \cdot \mathbf{u} / 2) \\
& +\bar{\rho}\left[\partial_{z} \hat{\mathbf{u}} \cdot \operatorname{DCT}(w \mathbf{u})-\hat{\mathbf{u}} \cdot \operatorname{DCT}\left(w \partial_{z} \mathbf{u}\right)\right] / 2 \\
& F_{h \uparrow}(\mathbf{k})=-\bar{\rho} \hat{\mathbf{u}} \cdot \operatorname{DCT}(w \mathbf{u}) / 2 \\
& F_{p \uparrow}(\mathbf{k})=-c_{p} \bar{\rho} \bar{\theta} \hat{w} \hat{\pi}^{\prime} \\
& C_{A \rightarrow h}(\mathbf{k})=c_{p} \bar{\rho} \bar{\theta} \hat{w} \partial_{z} \hat{\pi}^{\prime} \\
& \operatorname{Div}_{h}(\mathbf{k})=\bar{\rho} \hat{\mathbf{u}} \cdot \operatorname{DCT}\left[\mathbf{u}\left(\partial_{z} w+\nabla \cdot \mathbf{u}\right)\right] / 2 \\
& H_{h}(\mathbf{k})=c_{p} \bar{\rho} \hat{H}_{m} \hat{\pi}^{\prime} \\
& J_{h}(\mathbf{k})=-F_{h \uparrow}(\mathbf{k}) \partial_{z} \ln \bar{\rho} \\
& D_{h}(\mathbf{k})=\bar{\rho} \hat{\mathbf{u}} \cdot \hat{\mathcal{D}}_{\mathbf{u}}
\end{aligned}
$$

where $\mathrm{DCT}()$ is equivalent to ()$, \nabla$ is the horizontal gradient operator, $w$ is the vertical velocity, $c_{p}$ is the specific heat of dry air at constant pressure, $\bar{\theta}$ is the height-variant basic-state potential temperature; $\pi^{\prime}=\pi-\bar{\pi}=\left(p / p_{0}\right)^{R_{d} / c_{p}}-\left(\bar{p} / p_{0}\right)^{R_{d} / c_{p}}$ is the perturbed Exner pressure, with $p, p_{0}, \bar{p}$ and $R_{d}$ denoting pressure, reference surface pressure, basic-state pressure and gas constant for dry air, respectively; $H_{m}=\left(1+1.61 q_{v}\right) S_{\theta}+1.61 \theta S_{q v}$ is the combined diabatic contributions, with $q_{v}$ denoting water vapor mixing ratio, $\theta$ denoting potential temperature, and $S_{\theta}$ and $S_{q v}$ denoting the diabatic contributions to $d \theta / d t$ and $d q_{v} / d t$, respectively; and $\mathcal{D}_{\mathbf{u}}$ represents the dissipation of $\mathbf{u}$.

For the convenience of displaying the results, one-dimensional (1D) wavenumber spectra as a function of total horizontal wavenumber $k_{h}\left(=|\mathbf{k}|=\sqrt{k_{x}^{2}+k_{y}^{2}}\right)$ are constructed by angular averaging over the wavenumber band $k_{h}-\Delta k / 2 \leq|\mathbf{k}|<k_{h}+\Delta k / 2$ as

$$
\varepsilon\left(k_{h}\right)=\sum_{k_{h}-\Delta k / 2 \leq|\mathbf{k}|<k_{h}+\Delta k / 2} \varepsilon(\mathbf{k}) / \Delta k
$$

where $\varepsilon$ represents any one of the spectral terms in this study; $\Delta k=\pi /(\Delta N), \Delta$ is the horizontal grid spacing, $N=\min \left(N_{i}, N_{j}\right)$, and $N_{i}$ and $N_{j}$ are the number of grid points along the zonal and meridional directions, respectively. 


\subsection{Evolutions of the HKE Spectra}

Time evolutions of the vertically averaged HKE spectra over the troposphere $(z=0-12 \mathrm{~km})$ and lower stratosphere $(z=17-19 \mathrm{~km})$ are shown in Figures 3-5, respectively.

In the troposphere, it can be found from the distribution of HKE spectrum as a function of horizontal wavelength $\lambda_{h}$ (wavenumber $k_{h}$ ), which, with the decrease (increase) of $\lambda_{h}\left(k_{h}\right)$, the magnitude of HKE in general reduces sharply at the initial time (0000 UTC 6 August; Figure 3a), especially for $\lambda_{h}<200 \mathrm{~km}$ $\left(k_{h}>\pi \times 10^{-5} \mathrm{rad} \mathrm{m}^{-1}\right)$. It suggests that although the ECMWF ERA5 reanalysis data reach a horizontal resolution of $0.25^{\circ}$, the atmospheric information they contain at mesoscale is still limited. The model then takes about $6 \mathrm{~h}$ to complete the spinup adjustment, which reflects in the variation of the HKE spectrum. The spectrum experiences a significant lift for $\lambda_{h}<400 \mathrm{~km}\left(k_{h}>\pi / 2 \times 10^{-5} \mathrm{rad} \mathrm{m}^{-1}\right)$ during the first $3 \mathrm{~h}$ and a continued lift for $\lambda_{h}<80 \mathrm{~km}\left(k_{h}>\pi / 4 \times 10^{-4} \mathrm{rad} \mathrm{m}^{-1}\right)$ during the second $3 \mathrm{~h}$. Obviously, compared with larger scales, the spinup adjustment exerts more influence on $\lambda_{h}<100 \mathrm{~km}$ $\left(k_{h}>2 \pi \times 10^{-5} \mathrm{rad} \mathrm{m}^{-1}\right)$.

Accompanied with the intensification of Lekima, the HKE in the troposphere then increases at various horizontal scales in the following time (Figure $3 b-d$ ). Note that the HKE spectrum has already reproduced well the -3 slope at the synoptic scale and the $-5 / 3$ slope at the mesoscale of the real atmosphere just after the completion of spinup (0600 UTC 6 August; Figure 3a). Thus, it would have kept this canonical spectral shape if energy at various scales could increase comparatively in the log coordinate, whereas it is not the case here. The HKE spectrum can be roughly divided into three wavelength regions: $\lambda_{h}>300 \mathrm{~km}\left(k_{h}<2 \pi / 3 \times 10^{-5} \mathrm{rad} \mathrm{m}^{-1}\right), 100<\lambda_{h}<300 \mathrm{~km}$ $\left(2 \pi / 3 \times 10^{-5}<k_{h}<2 \pi \times 10^{-5} \mathrm{rad} \mathrm{m}^{-1}\right)$, and $\lambda_{h}<100 \mathrm{~km}\left(k_{h}>2 \pi \times 10^{-5} \mathrm{rad} \mathrm{m}^{-1}\right)$. For $\lambda_{h}>300 \mathrm{~km}$ $\left(k_{h}<2 \pi / 3 \times 10^{-5} \mathrm{rad} \mathrm{m}^{-1}\right)$ and $\lambda_{h}<100 \mathrm{~km}\left(k_{h}>2 \pi \times 10^{-5} \mathrm{rad} \mathrm{m}^{-1}\right)$, the spectrum in general appears as lifting consistently; thus, the spectral shape over this two wavelength regions remains stable. For $100<\lambda_{h}<300 \mathrm{~km}\left(2 \pi / 3 \times 10^{-5}<k_{h}<2 \pi \times 10^{-5} \mathrm{rad} \mathrm{m}^{-1}\right.$; see Figure 4 a-d for a zoomed-in view); however, the significant growth of energy firstly occurs at larger scales, and then extends to smaller scales. Consequently, the spectrum appears to be swelling during this wavelength region and shows an arc-like spectral shape.
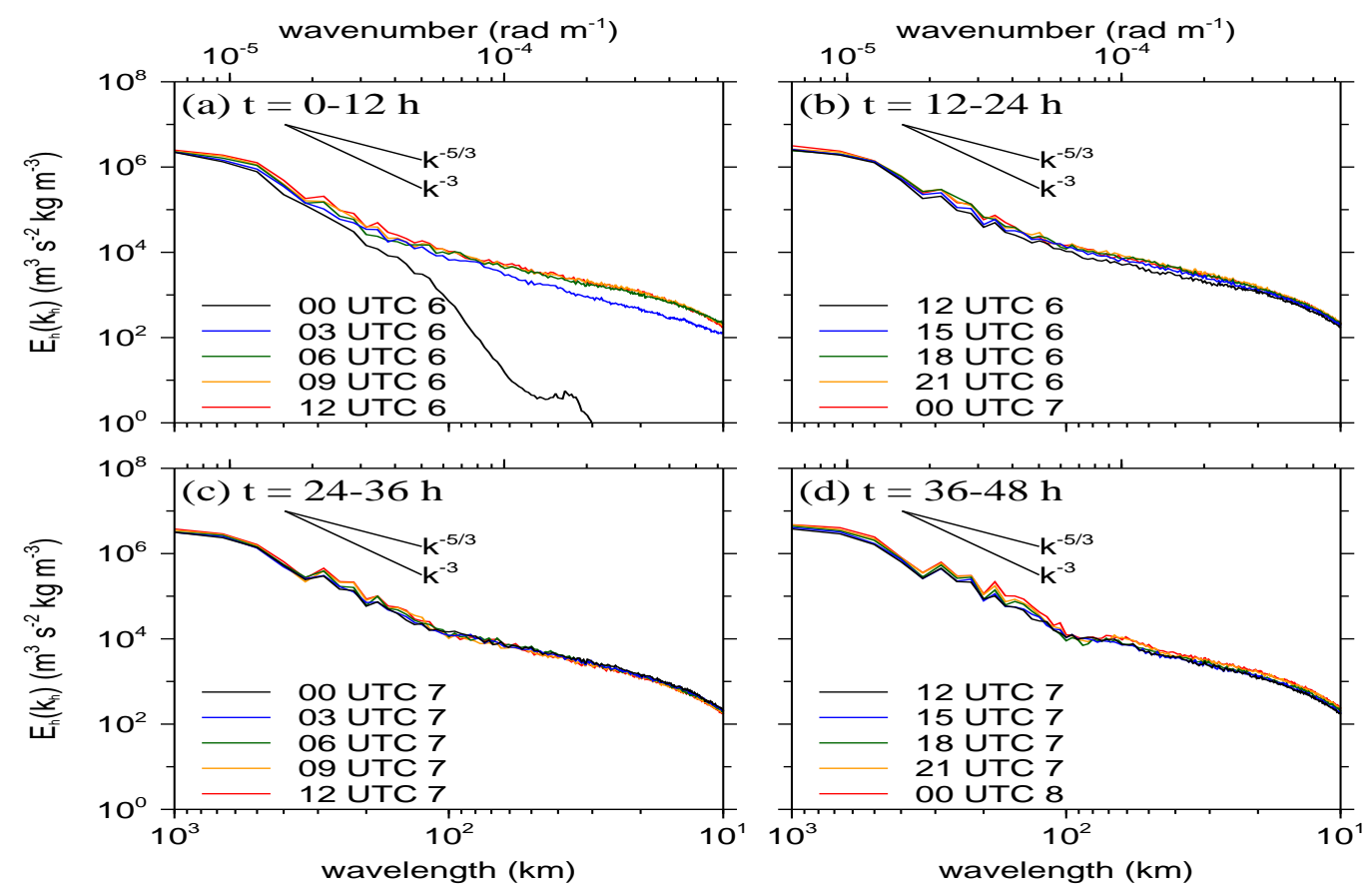

Figure 3. Time evolution of the horizontal kinetic energy (HKE) spectrum $\left[E_{h}\left(k_{h}\right)\right]$ averaged vertically over the troposphere for (a) 0-12 h, (b) 12-24 h, (c) 24-36 h, and (d) 36-48 h. Spectra are plotted every $3 \mathrm{~h}$. The reference lines correspond to the -3 and $-5 / 3$ slopes. 
The arc-like shape of HKE spectrum in the troposphere over the central mesoscale is an interesting phenomenon, which has also been revealed by the idealized TC simulation of WZ2018 and the observational hurricane study of Vonich and Hakim [32]. Beyond that, this phenomenon has not been identified by any other studies related to atmospheric energy spectrum. Therefore, it can be treated as a unique feature for TC. For the case in this study, it can be found from the evolution of the spectrum that before the intensification of Lekima, the HKE already possesses canonical spectrum, which is flat over the central mesoscale with a slope of approximately $-5 / 3$; while, with the rapid intensification of Lekima, the HKE spectrum gradually swells. In other words, the formation and development of this unique spectral feature is associated with the intensification of TC, which can be demonstrated by the observations. In Vonich and Hakim [32], they calculated the HKE spectra based on numbers of in situ observations and then classified the spectra according to storm intensity. Although they did not describe the hurricane spectra with expression similar to "arc-like shape" or "swelling", their conclusion is in essence consistent with ours. From their results (e.g., their Figure 7), the spectrum for tropical storms has a slope close to $-5 / 3$ over the central mesoscale. As the storm intensifies from category-1 to category-5, the swelling of the spectrum becomes increasingly obvious, which extends to increasingly smaller wavelengths. This leads them to the conclusion that "as storm intensity increases, spectral slope becomes steeper and the transition zone where spectral slope begins to steepen shifts downscale". The variation of their hurricane spectrum with storm intensity is in perfect agreement with the evolution of our TC spectrum during the intensification of Lekima. Note that in our simulation, Lekima reaches a maximum intensity of category-2 and the swelling of the spectrum extends to wavelengths (wavenumbers) around $100 \mathrm{~km}\left(2 \pi \times 10^{-5} \mathrm{rad} \mathrm{m}^{-1}\right)$; while in WZ2018, their idealized TC reaches a maximum intensity of category- 4 and the swelling extends to wavelengths (wavenumbers) around $50 \mathrm{~km}\left(2 \pi / 5 \times 10^{-4} \mathrm{rad} \mathrm{m}^{-1}\right)$. This also proves the relationship between TC intensification and the arc-like spectral shape.
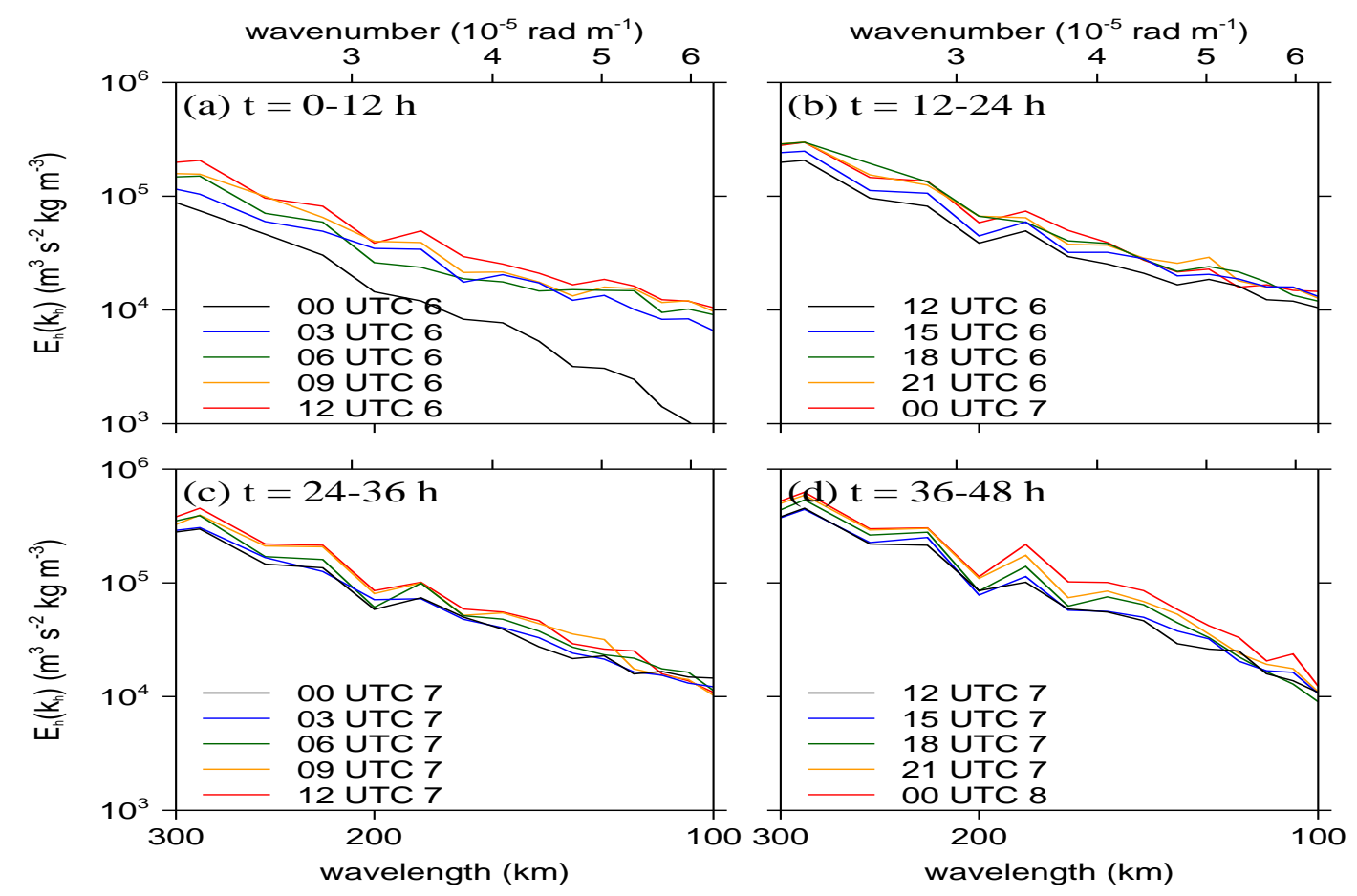

Figure 4. Time evolution of the HKE spectrum $\left[E_{h}\left(k_{h}\right)\right]$ averaged vertically over the troposphere for (a) 0-12 h, (b) 12-24 h, (c) 24-36 h, and (d) 36-48 h, and zoomed in for $100<\lambda_{h}<300 \mathrm{~km}\left(2 \pi / 3 \times 10^{-5}<\right.$ $\left.k_{h}<2 \pi \times 10^{-5} \mathrm{rad} \mathrm{m}^{-1}\right)$. Spectra are plotted every $3 \mathrm{~h}$. The reference lines correspond to the -3 and $-5 / 3$ slopes. 
Compared to that in the troposphere, the growth of HKE spectrum in the lower stratosphere shows different characteristics (Figure 5a-d). After the spinup adjustment of the model, the spectral shape keeps relatively stable throughout the intensification of Lekima. Differing from the canonical atmospheric spectrum, the lower-stratospheric HKE spectrum here has consistent $-5 / 3$ slope for $\lambda_{h}<1000 \mathrm{~km}\left(k_{h}>2 \pi \times 10^{-6} \mathrm{rad} \mathrm{m}^{-1}\right)$, but not -3 slope at the large-scale end. Meanwhile, the spectrum shows flat shape throughout the mesoscale but not arc-like shape over the central mesoscale as in the troposphere. These features are also identical to those found in WZ2018.
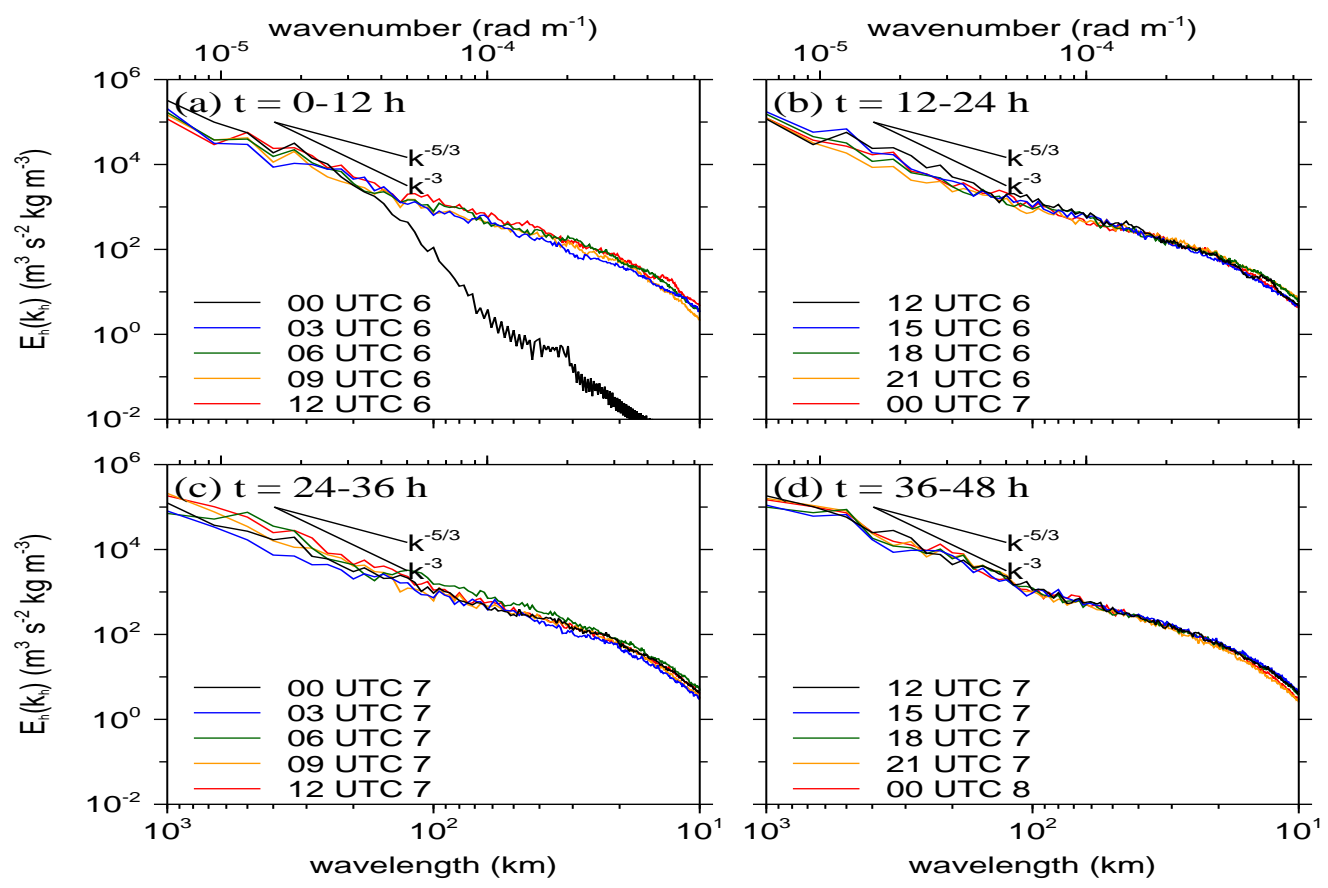

Figure 5. Time evolution of the HKE spectrum $\left[E_{h}\left(k_{h}\right)\right]$ averaged vertically over the lower stratosphere for (a) 0-12 h, (b) 12-24 h, (c) 24-36 h, and (d) 36-48 h. Spectra are plotted every $3 \mathrm{~h}$. The reference lines correspond to the -3 and $-5 / 3$ slopes.

To further understand the formation of the unique HKE spectra for TC, Figure 6 shows the rotational kinetic energy (RKE) and divergent kinetic energy (DKE) spectra at selected times. The RKE has spatiotemporal variable spectral shape. At 0600 UTC 6 August, the RKE spectrum in the troposphere is steeper at large scales and shallower at smaller scales, and the visible swelling of the spectrum appears for $200<\lambda_{h}<400 \mathrm{~km}\left(\pi / 2 \times 10^{-5}<k_{h}<\pi \times 10^{-5} \mathrm{rad} \mathrm{m}^{-1}\right.$; Figure 6a); while the RKE spectrum in the lower stratosphere is flat for $\lambda_{h}<1000 \mathrm{~km}\left(k_{h}>2 \pi \times 10^{-6} \mathrm{rad} \mathrm{m}^{-1}\right)$ with a slope approaching $-5 / 3$ (Figure 6b). After the intensification of Lekima at 0000 UTC 8 August, the swelling of the RKE in the troposphere is more significant, which extends downscale to $\lambda_{h}=100 \mathrm{~km}\left(k_{h}=2 \pi \times 10^{-5} \mathrm{rad} \mathrm{m}^{-1}\right.$; Figure 6c); while the lower-stratospheric RKE spectrum suffers much less change (Figure 6d). The DKE spectrum, in contrast, always shows a flat shape, which changes little with time and height. 

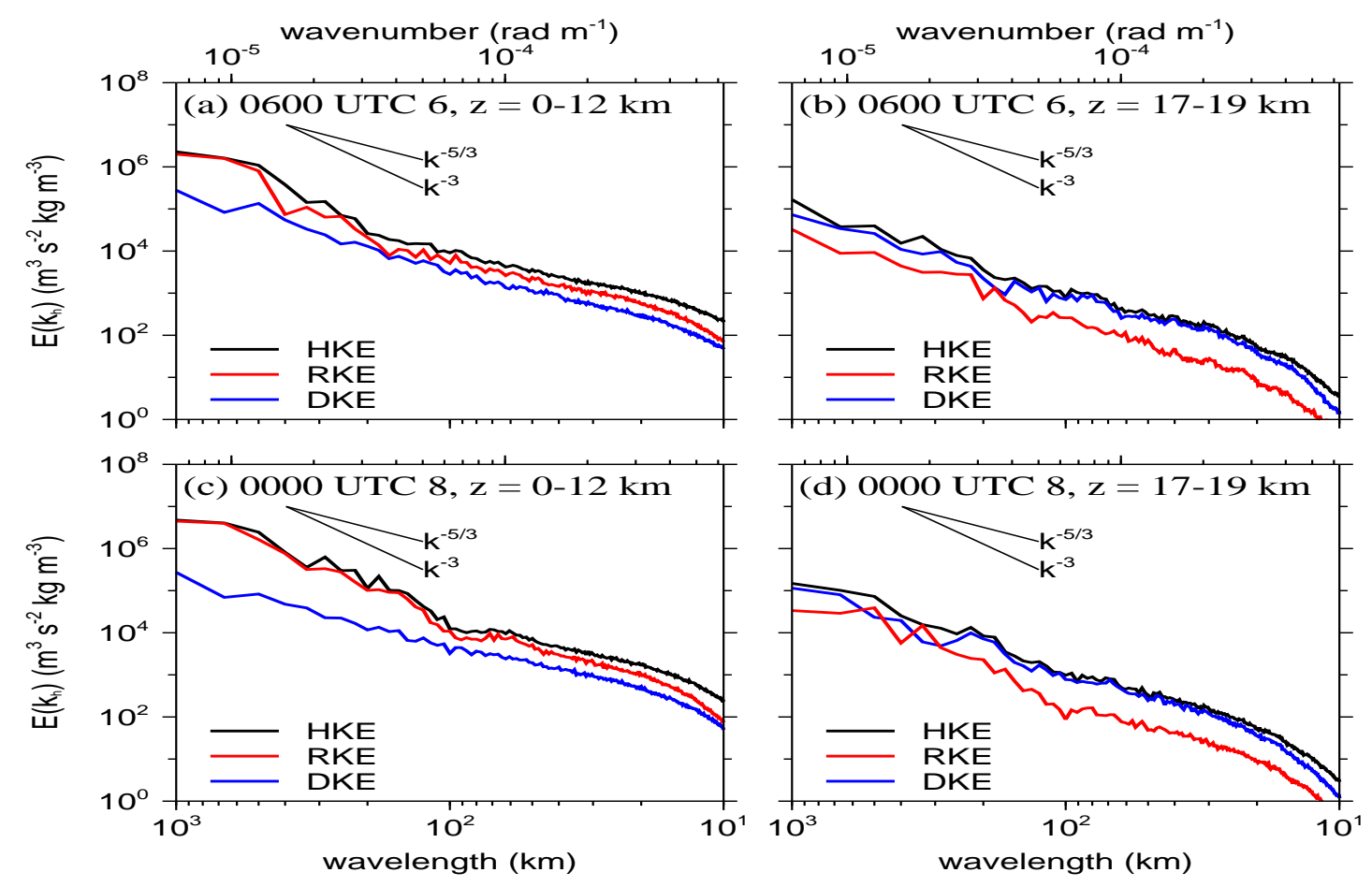

Figure 6. Horizontal wavenumber spectra of HKE (black line), rotational kinetic energy (RKE) (red line), and divergent kinetic energy (DKE) (blue line) averaged vertically over the (a,c) troposphere and $(\mathbf{b}, \mathbf{d})$ lower stratosphere, at (a,b) 0600 UTC 6 August and (c,d) 0000 UTC 8 August. The reference lines correspond to the -3 and $-5 / 3$ slopes.

From Figure 6, it is clear that in the troposphere, the HKE is dominated by the RKE over almost all wavelengths. Considering that the TC is characterized by intensive rotation, this is to be expected. Therefore, the tropospheric spectral shape of HKE is determined by the RKE spectrum to a large extent, and the gradual swelling of the HKE spectrum during the intensification of Lekima is induced by the variation of the RKE spectrum. This means that the unique arc-like spectrum for TC in the troposphere stems from the rotational mode of TC motion. When it comes to the lower stratosphere, the RKE spectrum becomes as flat as the DKE spectrum and the HKE tends to be dominated by the divergent mode, probably because that the vortex mode of the TC which is remarkable in the troposphere does not extend upward to higher heights, and the remnant of the TC outflow layer we examine here is strongly divergent. As both the RKE and the DKE have flat spectral shape with a slope of $-5 / 3$, the resultant lower-stratospheric HKE spectrum also inherits this feature.

\subsection{Spectral HKE Budget Diagnosis}

To determine the roles of different physical processes play during the intensification of Lekima, here we investigate the spectral budget of HKE using Equation (3). The 12-h period from 0000 UTC 7 August to 1200 UTC 7 is chosen for analysis. The earlier period is not chosen to avoid the impact of initial spinup adjustment of the model on the results. The later period is not chosen mainly for two reasons: (1) this period involves the mature stage of Lekima, and (2) Lekima in this period is too close to some islands, which leads to the local absence of physical quantities and affects the implementation of 2D DCT over the lower layers. Considering the particularity of the boundary layer and the vertical distribution of diabatic heating, the details of dynamics and energetics of HKE should vary with height in the troposphere. Thus, the troposphere is further divided into the lower troposphere $(z=0-1.5 \mathrm{~km})$, midtroposphere $(z=2.5-5 \mathrm{~km})$, and upper troposphere $(z=6-12 \mathrm{~km})$.

Figure 7 shows the various spectral budget terms averaged in time over the intensification of Lekima and vertically over different layers. For the energy conversion term $C_{A \rightarrow h}$ (black solid lines 
in the left panels of Figure 7), a positive value means a conversion induced by the buoyancy effect from APE to HKE and vice versa. In the lower troposphere, the magnitude of $C_{A \rightarrow h}$ is relatively weak at various horizontal scales (Figure 7a); while with the increase of height, the positive buoyancy dominates the troposphere and the importance of $C_{A \rightarrow h}$ becomes prominent. In the midtroposphere, except for sporadic scales such as wavelengths (wavenumbers) around 100 and $400 \mathrm{~km}\left(2 \pi \times 10^{-5}\right.$ and $\left.\pi / 2 \times 10^{-5} \mathrm{rad} \mathrm{m}^{-1}\right)$, it mainly makes a positive contribution, implying a dominant conversion from APE to HKE (Figure 7c). The sporadic negative contribution comes from the local-scale negative buoyancy forcing induced by the increase of air density, which originates from the evaporation and melting processes. In the upper troposphere, it becomes entirely positive, and develops two peaks at wavelengths (wavenumbers) of 300 and $1000 \mathrm{~km}\left(2 \pi / 3 \times 10^{-5}\right.$ and $\left.2 \pi \times 10^{-6} \mathrm{rad} \mathrm{m}^{-1}\right)$, respectively (Figure 7e). In general, a level of neutral buoyancy which is characterized as a transition zone from positive to negative buoyancy always exists near the tropopause. Thus, differing from the troposphere, the lower stratosphere is dominated by negative buoyancy forcing, and the $C_{A \rightarrow h}$ term always makes a negative contribution at all scales, implying a conversion from HKE to APE (Figure 7g).

The pressure vertical flux $F_{p \uparrow}$ corresponds to the flux of IGW energy at lowest order; thus, the divergence term $\partial_{z} F_{p \uparrow}$ can reflect the effect of vertical transportation of IGWs on the budget of HKE. The wavelength-height distribution of $F_{p \uparrow}$ is shown in Figure 8a, and note that a positive (negative) $F_{p \uparrow}$ means a downward (upward) flux. On the whole, $F_{p \uparrow}$ is upward in the lower stratosphere and downward in the troposphere at various scales, implying the domination of IGWs which occur in the upper troposphere and then propagate to higher and lower layers.

There are also nonnegligible differences at different scales. For $400<\lambda_{h}<1000 \mathrm{~km}\left(2 \pi \times 10^{-6}<k_{h}\right.$ $<\pi / 2 \times 10^{-5} \mathrm{rad} \mathrm{m}^{-1}$ ), the boundary between upward and downward $F_{p \uparrow}$ is around $z=15 \mathrm{~km}$; namely, the highest source region of the waves can reach the tropopause. With the reduction of height from 15 to $6 \mathrm{~km}$, the magnitude of $F_{p \uparrow}$ increases, which suggests that the downward-propagating waves are not only generated at the tropopause, but also generated throughout the upper troposphere. The $F_{p \uparrow}$ achieves its local maximum around $z=6 \mathrm{~km}$ and decreases from 6 to $4 \mathrm{~km}$, indicating that the IGW energy are deposited into this height range during the downward propagation of the waves. It then increases again and achieves another local maximum around $z=2.5 \mathrm{~km}$. Obviously, a secondary wave source exists in the midtroposphere for $z=2.5-4 \mathrm{~km}$. At lower heights, the $F_{p \uparrow}$ gradually decreases, and the IGW energy are powerfully deposited into the lower troposphere.

The vertical distribution of $F_{p \uparrow}$ for $\lambda_{h}<100 \mathrm{~km}\left(k_{h}>2 \pi \times 10^{-5} \mathrm{rad} \mathrm{m}^{-1}\right)$ is similar to that for $400<\lambda_{h}<1000 \mathrm{~km}\left(2 \pi \times 10^{-6}<k_{h}<\pi / 2 \times 10^{-5} \mathrm{rad} \mathrm{m}^{-1}\right)$, except that the boundary height between positive and negative $F_{p \uparrow}$ reduces to $z=13 \mathrm{~km}$; namely, the highest source region of the waves drops in height. For wavelengths (wavenumbers) slightly longer (smaller) than $100 \mathrm{~km}\left(2 \pi \times 10^{-5} \mathrm{rad} \mathrm{m}^{-1}\right)$; however, the distribution is not quite the same. Firstly, weak upward $F_{p \uparrow}$ appears for heights below $4 \mathrm{~km}$, which can be attributed to upward-propagating IGWs excited in the boundary layer. Secondly, the boundary between positive and negative $F_{p \uparrow}$ in the upper troposphere drops rapidly to $z=6 \mathrm{~km}$. In other words, the upper troposphere is not an effective source region for downward-propagating waves at these scales. 

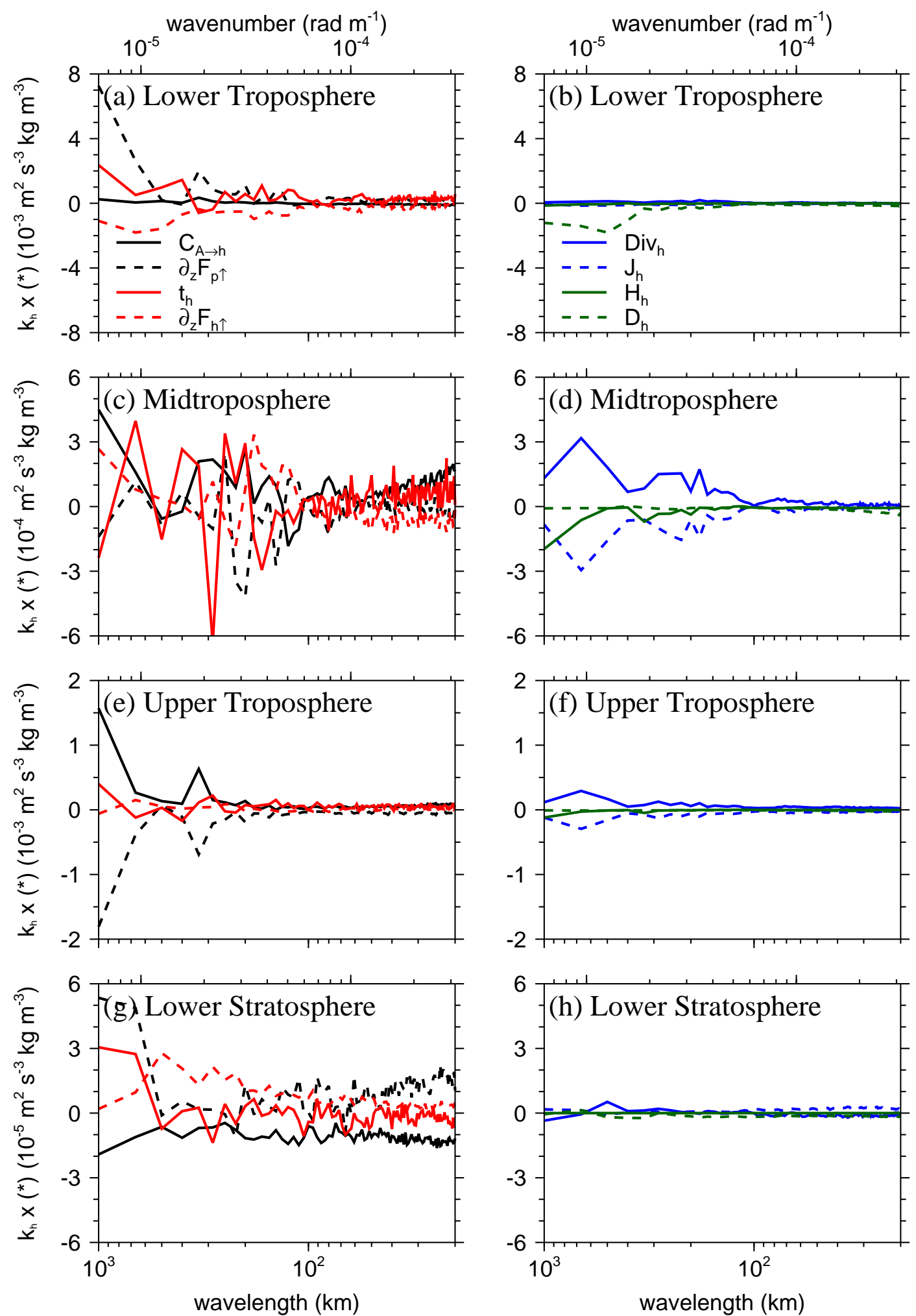

Figure 7. Spectral HKE budget terms averaged vertically over the $(\mathbf{a}, \mathbf{b})$ lower troposphere, $(\mathbf{c}, \mathbf{d})$ midtroposphere, $(\mathbf{e}, \mathbf{f})$ upper troposphere, and $(\mathbf{g}, \mathbf{h})$ lower stratosphere, and in time over the intensification period. The legend for the left panels is given in (a) and that for the right panels in (b). The asterisk * represents any one of the budget terms. The spectra are multiplied by $k_{h}$ to preserve area in log-linear coordinates. 

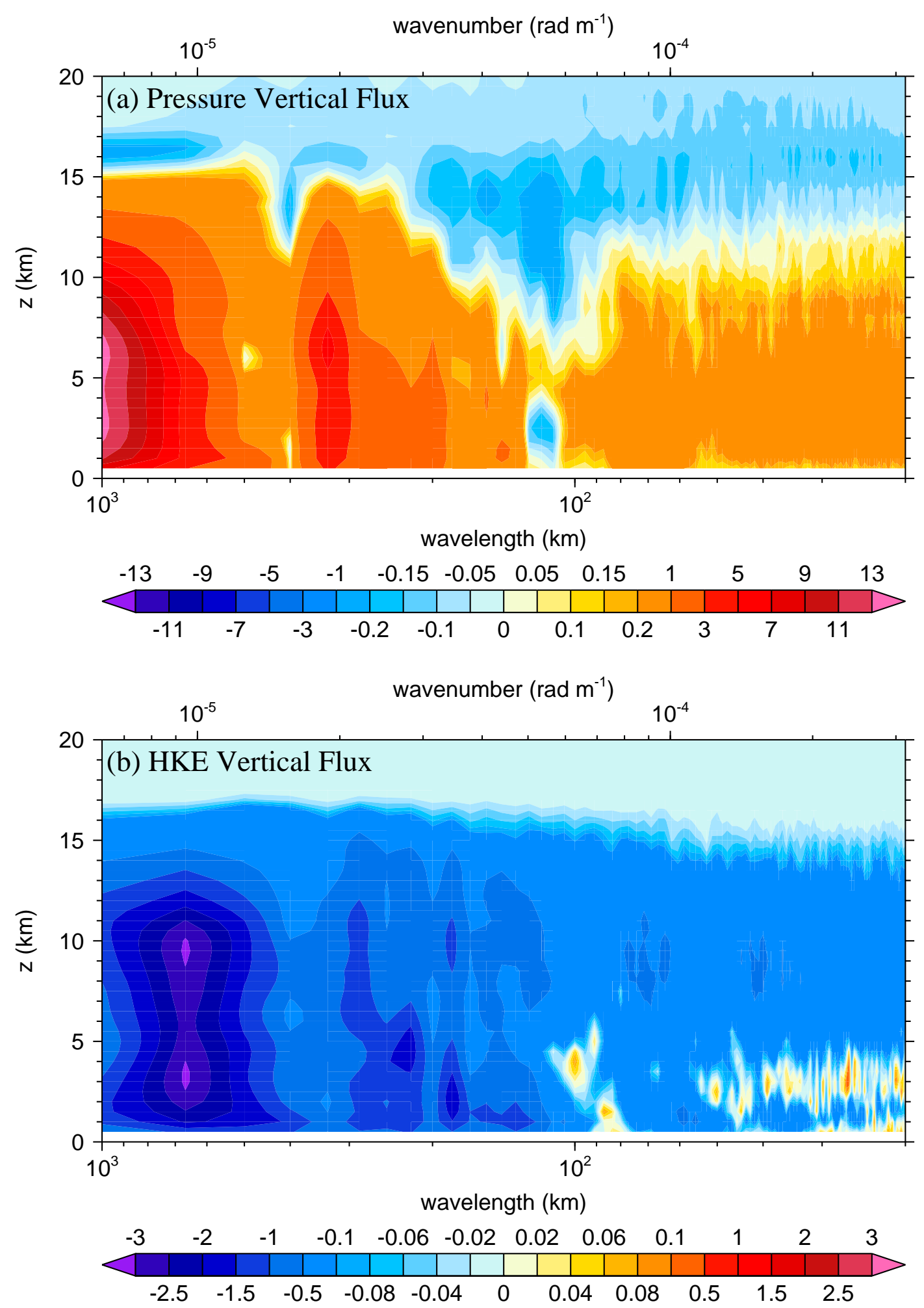

Figure 8. Horizontal wavelength-height distribution of (a) pressure vertical flux and (b) HKE vertical flux averaged in time over the intensification period $\left(\mathrm{m}^{2} \mathrm{~s}^{-3} \mathrm{~kg} \mathrm{~m}^{-2}\right)$.

Although there is weak upward $F_{p \uparrow}$ at wavelengths (wavenumbers) slightly greater (smaller) than $100 \mathrm{~km}\left(2 \pi \times 10^{-5} \mathrm{rad} \mathrm{m}^{-1}\right)$, the lower troposphere is primarily the net inflow layer of downward $F_{p \uparrow}$. Meanwhile, the upper troposphere is always the net outflow layer of $F_{p \uparrow}$ and the lower stratosphere is the net inflow layer of upward $F_{p \uparrow}$. Consequently, the $\partial_{z} F_{p \uparrow}$ term (black shaded lines in the left panels of 
Figure 7) makes clear positive, negative, and positive contribution in the lower troposphere (Figure 7a), upper troposphere (Figure 7e), and lower stratosphere (Figure 7g), respectively. The midtroposphere, however, is both the inflow and the outflow layer of $F_{p \uparrow}$, depending on the wavelength; thus the contribution of $\partial_{z} F_{p \uparrow}$ with wavelength over this layer is complicated (Figure 7c).

The spectral transfer term $t_{h}$ (red solid lines in the left panels of Figure 7) represents the transfer of HKE among different horizontal scales, which is caused by nonlinear interactions. It actually reflects the role of the so called "energy cascade" on budget of HKE at different scales. By constructing nonlinear spectral flux of HKE using $t_{h}$, one can identify the direction of energy cascade, which is a key topic of research on atmospheric energy (WZ2018). In this paper, however, as the limited-area domain we analyze has open lateral boundaries, the spectral flux integrated over the whole domain is not equal to zero, and the direction of energy cascade cannot be identified accurately; thus, we only discuss the distribution of $t_{h}$. In the lower troposphere, the energy cascade makes a negative contribution at wavelengths (wavenumbers) around $300 \mathrm{~km}\left(2 \pi / 3 \times 10^{-5} \mathrm{rad} \mathrm{m}^{-1}\right)$ and makes a positive contribution for the rest scales (Figure 7a). In the midtroposphere, the effect of energy cascade is complicated and $t_{h}$ crosses zero several times with the variation of wavelength. However, it makes a positive contribution at most scales (Figure 7c). When it comes to the upper troposphere, the role of $t_{h}$ is weak, with negative contribution for $400<\lambda_{h}<800 \mathrm{~km}\left(\pi / 4 \times 10^{-5}<k_{h}<\pi / 2 \times 10^{-5} \mathrm{rad} \mathrm{m}^{-1}\right)$ and $200<\lambda_{h}<300 \mathrm{~km}$ $\left(2 \pi / 3 \times 10^{-5}<k_{h}<2 \pi \times 10^{-5} \mathrm{rad} \mathrm{m}^{-1}\right)$, and positive contribution for the remainder (Figure 7e). The distribution of $t_{h}$ is relatively clear in the lower stratosphere, which contributes positively for $\lambda_{h}>500 \mathrm{~km}\left(k_{h}<2 \pi / 5 \times 10^{-5} \mathrm{rad} \mathrm{m}^{-1}\right)$ and negatively at smaller scales (Figure $7 \mathrm{~g}$ ).

The $F_{h \uparrow}$ term is the HKE vertical flux, and the corresponding divergence term $\partial_{z} F_{h \uparrow}$ reflects the direct contribution of vertical transportation of convection to HKE. It can be found from Figure $8 \mathrm{~b}$ that, for most scales, the $F_{h \uparrow}$ is consistently upward from the lower troposphere to the lower stratosphere, which suggests that convection within the troposphere transports the energy upward. Two prominent subranges of $F_{h \uparrow}$ are collocated with those of the diabatic heating (Figure 2b), implying the close connection between the convection intensity and release of heating. There is also sporadic weak downward $F_{h \uparrow}$ in the lower troposphere and midtroposphere for wavelengths (wavenumbers) around $100 \mathrm{~km}\left(2 \pi \times 10^{-5} \mathrm{rad} \mathrm{m}^{-1}\right)$ and below $60 \mathrm{~km}$ (above $\left.\pi / 3 \times 10^{-4} \mathrm{rad} \mathrm{m}^{-1}\right)$, which is likely to be associated with the evaporation and the drag effect of precipitation particles. Reflected in the budget of HKE, the $\partial_{z} F_{h \uparrow}$ term (red shaded lines in the left panels of Figure 7) in general makes a negative contribution in the lower troposphere (Figure 7a) and a positive contribution over the higher layers (Figure 7c,e,g).

The 3D divergence term $\operatorname{Div}_{h}$ and adiabatic nonconservation term $J_{h}$ (blue solid and dashed lines in the right panels of Figure 7, respectively) stem from the compressibility and adiabatic nonconservation of the atmosphere, respectively. They are both significant in the midtroposphere and upper troposphere, and always play the balanced positive and negative contributions (Figure $7 \mathrm{~d}, \mathrm{f}$ ).

The diabatic term $H_{h}$ (green solid lines in the right panels of Figure 7) represents the direct effect of diabatic processes on HKE, which is only relatively remarkable in the midtroposphere with negative contribution (Figure 7d).

The dissipative term $D_{h}$ (green dashed lines in the right panels of Figure 7) represents the effect of dissipative processes. Because of boundary-layer friction, its role is significant in the lower troposphere, with large negative contribution for $\lambda_{h}>300 \mathrm{~km}\left(k_{h}<2 \pi / 3 \times 10^{-5} \mathrm{rad} \mathrm{m}^{-1}\right.$; Figure $\left.7 \mathrm{~b}\right)$. However, for higher layers, it plays weak role (Figure $7 \mathrm{~d}, \mathrm{f}, \mathrm{h}$ ).

Overall, despite differences in magnitude and specific scales the effect are exerted on, the contributions of various budget terms at different heights during the intensification of Lekima are qualitatively consistent with those of the idealized TC in WZ2018, which suggests that the roles of various physical processes play are relatively steady. The spectral transfer term is an exception, which shows more complicated distribution here than that in WZ2018, implying the complexity of energy cascade associated with TC in real atmosphere.

During the intensification of Lekima, according to the $C_{A \rightarrow h}$ term, the system is in general baroclinically unstable in the troposphere and stable in the lower stratosphere. Due to the 
indetermination of the direction of energy cascade, the barotropic instability associated with the $t_{h}$ term cannot be determined. However, by comparing the magnitude of $C_{A \rightarrow h}$ and $t_{h}$, the barotropic process dominates the baroclinic process in the lower troposphere, and the opposite happens in the upper troposphere; while these two processes are comparable in the midtroposphere. In other words, with the increase of height in the troposphere, the relative importance of barotropic process weakens, while the importance of baroclinic process enhances. Based on a forced shallow-water model and primitive equation model, respectively, Hendricks et al. [33] and Williams [34] discussed the potential vorticity dynamics associated with $\mathrm{TC}$, and found the importance of barotropic instability during the evolution of TC inner region. Although the focuses of their research and the models and methods they used were all different from us in this paper, their conclusions on barotropic and baroclinic processes were essentially consistent with the results here, which supports our finding from a more theoretical perspective.

In addition, note that the various terms in the troposphere are stagnant for $\lambda_{h}<100 \mathrm{~km}$ $\left(k_{h}>2 \pi \times 10^{-5} \mathrm{rad} \mathrm{m}^{-1}\right)$ compared with those at larger scales. On the one hand, this matches the fact that the swelling of HKE spectrum only extends downscale to wavelengths (wavenumbers) around $100 \mathrm{~km}\left(2 \pi \times 10^{-5} \mathrm{rad} \mathrm{m}^{-1}\right)$. On the other hand, it implies that the activation of energy activity at smaller scales is the key to the downscale extension of the arc-like spectral shape with the intensification of TC.

\section{Discussion and Conclusions}

In this paper, the intensification of Typhoon Lekima is simulated for the purpose of investigating the evolution and characteristics of HKE spectra and diagnosing the corresponding spectral HKE budgets for real TC.

Accompanied with the intensification of Lekima, the HKE spectra at various heights vary remarkably. After the completion of the intensification, the spectra reproduce the canonical atmospheric spectrum to some extent, but also exhibit significant differences from the canonical spectrum. In the troposphere, the spectrum is in perfect agreement with the canonical spectrum before the intensification of Lekima; while accompanied with the intensification, the spectrum is gradually modified, and an arc-like spectral shape forms over the central mesoscale and extends to smaller scales. The investigation on RKE and DKE shows that the appearance of the arc-like shape is a direct result of the increasing vorticity during the intensification of Lekima. Combined with the idealized TC simulation of WZ2018 and the observational hurricane study of Vonich and Hakim [32], this arc-like shape is a unique and robust feature for atmospheric HKE spectrum associated with TC. The stronger the TC, the more pronounced this feature is and the smaller scale it extends to. In the lower stratosphere, the spectrum is in perfect agreement with the canonical spectrum at the mesoscale with a slope of $-5 / 3$, but the -3 slope at the synoptic scale does not appear.

The canonical atmospheric spectrum is obtained by averaging numerous of observations, and is considered representative and universal to the earth's atmosphere. However, the results in this study demonstrate that the canonical spectrum does not always apply to all cases. In fact, the evolution of HKE spectrum here in the troposphere directly reflects the modification of the spectrum by the development of Lekima. It is thus a reasonable inference that weather systems with unique structure and vibrant energy activity have the potential of modifying the canonical atmospheric spectrum during their development and evolution. Future investigations on the atmospheric energy spectrum should focus upon the spectral feature associated with specific weather systems, and seek to determine the formation mechanism behind the potential difference from the canonical spectrum. For example, why does the enhancement of rotational mode of TC lead to the swelling of HKE spectrum over the central mesoscale?

The predictability of TC indicated by the energy spectra, which has not been discussed here, also deserves to be explored. According to Lorenz [35], atmospheric system governed by a $k^{-p}$ power law has limited predictability when $p<3$ and unlimited predictability when $p \geq 3$. The arc-like 
shape of HKE spectrum over the central mesoscale means that $p$ for TC system changes dramatically over a relatively narrow scale range, which may imply that the predictability of TC is complicated at the mesoscale.

By diagnosing the spectral HKE budget, the roles various physical processes play during the intensification of Lekima are investigated. Some processes, such as the buoyancy forcing, vertical transportation of IGWs, nonlinear interactions, and vertical transportation of convection, make remarkable contributions at almost all heights from lower troposphere to lower stratosphere. Other processes, by contrast, only make significant contributions at specific heights. The results for various budget terms here are qualitatively consistent with those in WZ2018, which both verifies the reasonability and authenticity of results from the idealized TC simulation and also suggests that the roles of various physical processes play during the intensification of TC are relatively steady.

The dependence of budget terms on scale and height is intriguing. For example, from the pressure vertical flux and its divergence, it can be found that upward- and downward-propagating IGWs with broad horizontal-wavelength range are excited near the tropopause. This phenomenon is consistent with the description of mechanical oscillator mechanism, which has been proposed to account for the generation of gravity waves excited by convective systems [36,37]. Meanwhile, strong generation sources of IGWs also exist in the upper troposphere and midtroposphere. Note that the two maximum centers of pressure vertical flux (Figure 8a) are right at the bottom of the two diabatic heating subranges (Figure 2b); thus, it is reasonable to relate the generation of IGWs in the upper troposphere and midtroposphere with the forcing of heating [38]. Other interesting phenomena about IGWs are also worth noting: the specific height where the waves are generated near the tropopause is different for waves with different scales; for waves with wavelengths (wavenumbers) between 90 and $200 \mathrm{~km}$ $\left(2 \pi / 9 \times 10^{-4}\right.$ and $\left.\pi \times 10^{-5} \mathrm{rad} \mathrm{m}^{-1}\right)$, the tropopause and upper troposphere are even not effective emission layers; and the upward-propagating waves in the lower troposphere are only remarkable for wavelengths (wavenumbers) slightly larger (smaller) than $100 \mathrm{~km}\left(2 \pi \times 10^{-5} \mathrm{rad} \mathrm{m}^{-1}\right)$. The selectivity of the generated IGWs on height and horizontal scale deserves further confirmation and study.

In addition, why does sporadic negative buoyancy forcing in the midtroposphere only appear at wavelengths (wavenumbers) around 100 and $400 \mathrm{~km}\left(2 \pi \times 10^{-5}\right.$ and $\left.\pi / 2 \times 10^{-5} \mathrm{rad} \mathrm{m}^{-1}\right)$ ? What makes the effect of nonlinear interactions so complicated in the troposphere for TC in the real atmosphere? And what determines the downward HKE vertical flux associated with the drag effect of precipitation particles at wavelengths (wavenumbers) around $100 \mathrm{~km}\left(2 \pi \times 10^{-5} \mathrm{rad} \mathrm{m}^{-1}\right)$ and below $60 \mathrm{~km}$ (above $\pi / 3 \times 10^{-4} \mathrm{rad} \mathrm{m}^{-1}$ ) ? These problems referring to selectivity on scale are also under explored.

For our simulation, the swelling of the HKE spectrum in the troposphere just extends to wavelengths (wavenumbers) around $100 \mathrm{~km}\left(2 \pi \times 10^{-5} \mathrm{rad} \mathrm{m}^{-1}\right)$; meanwhile, the vibrant spectral HKE budgets are restricted to wavelengths larger than $100 \mathrm{~km}$. It is thus reasonable to connect the intensification of TC, the activation of energy activity at smaller scales, and the downscale extension of the arc-like spectral shape. The details and mechanisms are still unclear, pending further investigation.

Author Contributions: Conceptualization, Y.Z. and Y.W.; formal analysis, H.Z.; methodology, L.Z. and J.P.; software, S.L. and A.L.; writing-original draft, H.Z.; and writing-review and editing, Y.Z. and Y.W. All authors have read and agreed to the published version of the manuscript.

Funding: This research was funded by the National Natural Science Foundation of China, grant number 41975066; the Beijing Open Research Fund for Nanjing Joint Center of Atmospheric Research, grant number NJCAR2018ZD03; the National Natural Science Foundation for Young Scientists of China, grant number 41705037 and 41805092.

Conflicts of Interest: The authors declare no conflict of interest. 


\section{References}

1. Cangialosi, J.P.; Franklin, J.L. National Hurricane Center Verification Report. Tropical Prediction Center, National Hurricane Center, National Center for Environmental Prediction, National Weather Center, NOAA: Silver Spring, MD, USA, 2012; p. 76. Available online: https://www.nhc.noaa.gov/verification/pdfs/ Verification_2011.pdf (accessed on 2 July 2020).

2. Elsberry, R.L.; Lambert, T.D.B.; Boothe, M.A. Accuracy of Atlantic and eastern North Pacific tropical cyclone intensity forecast guidance. Weather Forecast. 2007, 22, 747-762. [CrossRef]

3. Zhang, F.; Tao, D. Effects of vertical wind shear on the predictability of tropical cyclones. J. Atmos. Sci. 2013, 70, 975-983. [CrossRef]

4. Qin, N.; Zhang, D.-L.; Miller, W.; Kieu, C.Q. On the rapid intensification of Hurricane Wilma (2005). Part IV: Inner-core dynamics during the steady radius of maximum wind stage. Q. J. R. Meteorol. Soc. 2018, 144, 2508-2523. [CrossRef]

5. Chen, X.; Zhang, J.A.; Marks, F.D. A thermodynamic pathway leading to rapid intensification of tropical cyclones in shear. Geophys. Res. Lett. 2019, 46, 9241-9251. [CrossRef]

6. Nastrom, G.D.; Gage, K.S. A climatology of atmospheric wavenumber spectra observed by commercial aircraft. J. Atmos. Sci. 1985, 42, 950-960. [CrossRef]

7. Cho, J.; Anderson, B.E.; Barrick, J.D.; Gregory, G.L.; Sachse, G.W.; Carroll, M.A.; Albercook, G.M.; Zhu, Y.; Newell, R.E. Horizontal wavenumber spectra of winds, temperature, and trace gases during the Pacific Exploratory Missions: 1. Climatology. J. Geophys. Res. 1999, 104, 5697-5716. [CrossRef]

8. Lindborg, E. Can the atmospheric kinetic energy spectrum be explained by two-dimensional turbulence? J. Fluid Mech. 1999, 388, 259-288. [CrossRef]

9. Dewan, E.M. Stratospheric wave spectra resembling turbulence. Science 1979, 204, 832-835. [CrossRef]

10. Gage, K.S. Evidence for a $k^{-5 / 3}$ law inertial range in mesoscale two-dimensional turbulence. J. Atmos. Sci. 1979, 36, 1950-1954. [CrossRef]

11. Tung, K.K.; Orlando, W.W. The $k^{-3}$ and $k^{-5 / 3}$ energy spectrum of atmospheric turbulence: Quasigeostrophic two-level model simulation. J. Atmos. Sci. 2003, 60, 824-835. [CrossRef]

12. Tulloch, R.; Smith, K.S. A theory for the atmospheric energy spectrum: Depth-limited temperature anomalies at the tropopause. Proc. Natl. Acad. Sci. USA 2006, 103, 14690-14694. [CrossRef] [PubMed]

13. Skamarock, W.C. Evaluating mesoscale NWP models using kinetic energy spectra. Mon. Weather Rev. 2004, 132, 3019-3032. [CrossRef]

14. Takahashi, Y.O.; Hamilton, K.; Ohfuchi, W. Explicit global simulations of the mesoscale spectrum of atmospheric motions. Geophys. Res. Lett. 2006, 33, 12812. [CrossRef]

15. Hamilton, K.; Takahashi, Y.O.; Ohfuchi, W. The mesoscale spectrum of atmospheric motions investigated in a very fine resolution global general circulation model. J. Geophys. Res. 2008, 113, 18110. [CrossRef]

16. Skamarock, W.C.; Park, S.-H.; Klemp, J.B.; Snyder, C. Atmospheric kinetic energy spectra from global high-resolution nonhydrostatic simulations. J. Atmos. Sci. 2014, 71, 4369-4381. [CrossRef]

17. Koshyk, J.N.; Hamilton, K. The horizontal kinetic energy spectrum and spectral budget simulated by a high-resolution troposphere-stratosphere-mesosphere GCM. J. Atmos. Sci. 2001, 58, 329-348. [CrossRef]

18. Waite, M.L.; Snyder, C. The mesoscale kinetic energy spectrum of a baroclinic life cycle. J. Atmos. Sci. 2009, 66, 883-901. [CrossRef]

19. Waite, M.L.; Snyder, C. Mesoscale energy spectra of moist baroclinic waves. J. Atmos. Sci. 2013, 70, $1242-1256$. [CrossRef]

20. Peng, J.; Zhang, L.; Luo, Y.; Zhang, Y. Mesoscale energy spectra of the mei-yu front system. Part I: Kinetic energy spectra. J. Atmos. Sci. 2014, 71,37-55. [CrossRef]

21. Peng, J.; Zhang, L.; Luo, Y.; Xiong, C. Mesoscale energy spectra of the mei-yu front system. Part II: Moist available potential energy spectra. J. Atmos. Sci. 2014, 71, 1410-1424. [CrossRef]

22. Wang, Y.; Zhang, L.; Peng, J.; Liu, S. Mesoscale horizontal kinetic energy spectra of a tropical cyclone. J. Atmos. Sci. 2018, 75, 3579-3596. [CrossRef]

23. Wang, Y.; Zhang, L.; Peng, J.; Zhang, Y.; Wei, T. Investigation of mesoscale available potential energy spectra in a simulated tropical cyclone. J. Meteorol. Res. 2019, 33, 1098-1112. [CrossRef] 
24. Skamarock, W.C.; Klemp, J.B.; Dudhia, J.; Gill, D.O.; Barker, D.M.; Wang, W.; Powers, J.G. A Description of the Advanced Research WRF Version 3. NCAR Tech. Note NCAR/TN-4751STR. 2008, p. 13. Available online: http://citeseerx.ist.psu.edu/viewdoc/summary?doi=10.1.1.484.3656 (accessed on 6 July 2020).

25. Thompson, G.; Field, P.R.; Rasmussen, R.M.; Hall, W.D. Explicit forecasts of winter precipitation using an improved bulk microphysics scheme. Part II: Implementation of a new snow parameterization. Mon. Weather Rev. 2008, 136, 5095-5115. [CrossRef]

26. Hong, S.-Y.; Noh, Y.; Dudhia, J. A new vertical diffusion package with an explicit treatment of entrainment processes. Mon. Weather Rev. 2006, 134, 2318-2341. [CrossRef]

27. Iacono, M.J.; Delamere, J.S.; Mlawer, E.J.; Shephard, M.W.; Clough, S.A.; Collins, W.D. Radiative forcing by long-lived greenhouse gases: Calculations with the AER radiative transfer models. J. Geophys. Res. 2008, 113, 13103. [CrossRef]

28. Klemp, J.B.; Dudhia, J.; Hassiotis, A.D. An upper gravity-wave absorbing layer for NWP applications. Mon. Weather Rev. 2008, 136, 3987-4004. [CrossRef]

29. Kain, J.S. The kain-fritsch convective parameterization: An update. J. Appl. Meteorol. 2004, 43, $170-181$. [CrossRef]

30. Denis, B.; Côté, J.; Laprise, R. Spectral decomposition of two-dimensional atmospheric fields on limited-area domains using the discrete cosine transform (DCT). Mon. Weather Rev. 2002, 130, 1812-1829. [CrossRef]

31. Peng, J.; Zhang, L.; Guan, J. Applications of a moist nonhydrostatic formulation of the spectral energy budget to baroclinic waves. Part I: The lower-stratospheric energy spectra. J. Atmos. Sci. 2015, 72, 2090-2108. [CrossRef]

32. Vonich, P.T.; Hakim, G.J. Hurricane kinetic energy spectra from in situ aircraft observations. J. Atmos. Sci. 2018, 75, 2523-2532. [CrossRef]

33. Hendricks, E.A.; Schubert, W.H.; Chen, Y.-H.; Kuo, H.-C.; Peng, M.S. Hurricane eyewall evolution in a forced shallow-water model. J. Atmos. Sci. 2014, 71, 1623-1643. [CrossRef]

34. Williams, G.J. The generation and maintenance of hollow PV towers in a forced primitive equation model. Meteorol. Atmos. Phys. 2019, 131, 1635-1659. [CrossRef]

35. Lorenz, E.N. The predictability of a flow which possesses many scales of motion. Tellus 1969, 21, $289-307$. [CrossRef]

36. Lane, T.P.; Reeder, M.J.; Clark, T.L. Numerical modeling of gravity wave generation by deep tropical convection. J. Atmos. Sci. 2001, 58, 1249-1274. [CrossRef]

37. Wang, Y.; Zhang, L.; Peng, J.; Guan, J. Mesoscale gravity waves in the mei-yu front system. J. Atmos. Sci. 2018, 75, 587-609. [CrossRef]

38. Baik, J.-J.; Hwang, H.-S.; Chun, H.-Y. Transient, linear dynamics of a stably stratified shear flow with thermal forcing and a critical level. J. Atmos. Sci. 1999, 56, 483-499. [CrossRef] 